Document downloaded from:

http://hdl.handle.net/10251/162257

This paper must be cited as:

Woodruff, K.; Baeza-Rubio, J.; Huerta, D.; Jones, BJP.; Mcdonald, AD.; Norman, L.; Nygren, DR.... (2020). Radio Frequency and DC High Voltage Breakdown of High Pressure Helium, Argon, and Xenon. Journal of Instrumentation. 15(4):1-15. https://doi.org/10.1088/17480221/15/04/P04022

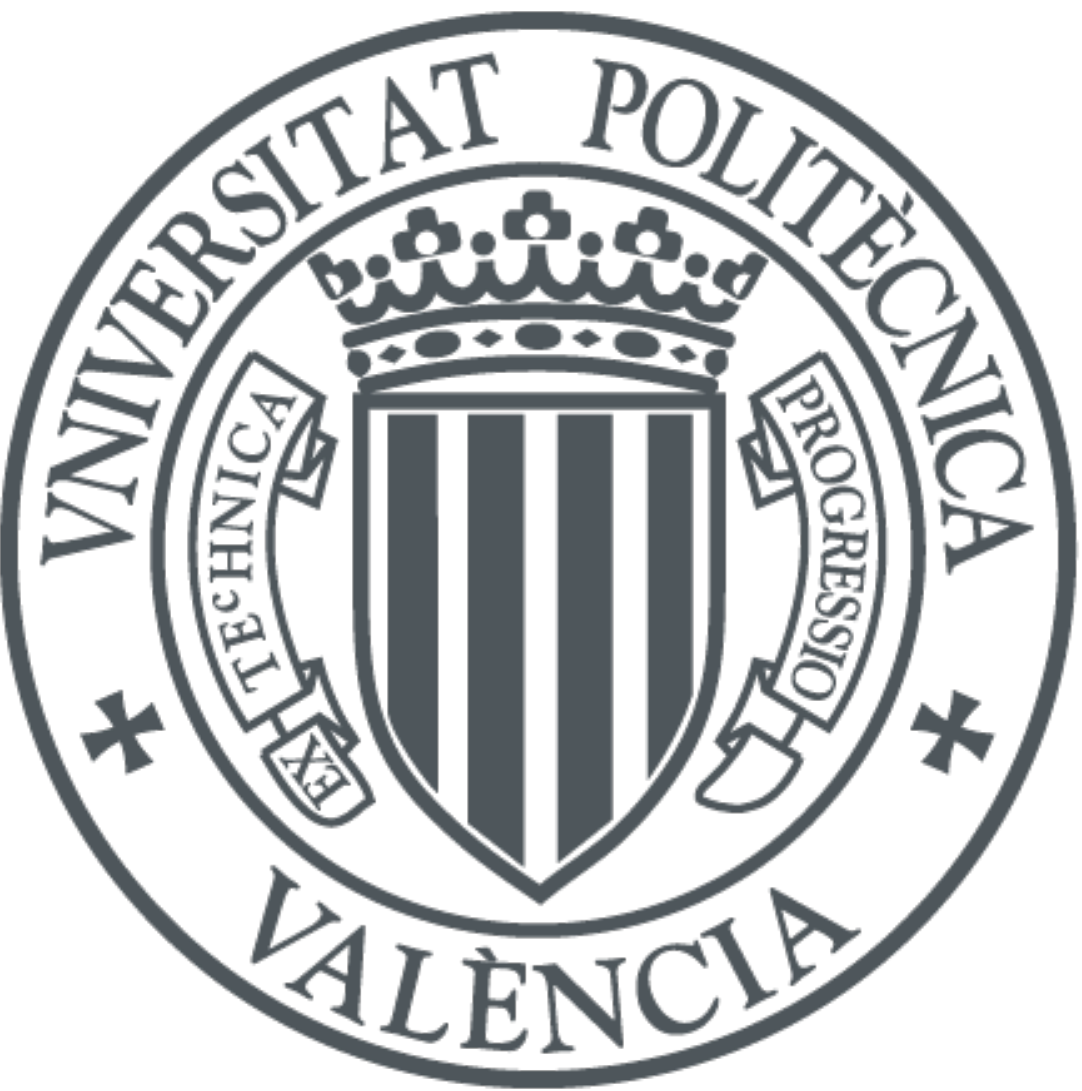

The final publication is available at

https://doi.org/10.1088/1748-0221/15/04/P04022

Copyright IOP Publishing

Additional Information 


\section{Radio Frequency and DC High Voltage Breakdown of High Pressure Helium, Argon, and Xenon}

\section{The NEXT Collaboration}

K. Woodruff, ${ }^{1, a}$ J. Baeza-Rubio, ${ }^{1}$ D. Huerta, ${ }^{1}$ B. J. P. Jones, ${ }^{1}$ A. D. McDonald, ${ }^{1}$ L. Norman, ${ }^{1}$ D. R. Nygren, ${ }^{1}$ C. Adams, ${ }^{10}$ V. Álvarez, ${ }^{17}$ L. Arazi,,${ }^{6}$ I.J. Arnquist, ${ }^{18}$ C.D.R Azevedo, ${ }^{4}$ K. Bailey, ${ }^{19}$ F. Ballester, ${ }^{20}$ J.M. Benlloch-Rodríguez, ${ }^{17}$ F.I.G.M. Borges, ${ }^{12}$ N.K. Byrnes, ${ }^{1}$ S. Cárcel, ${ }^{17}$ J.V. Carrión, ${ }^{17}$ S. Cebrián, ${ }^{21}$ E. Church, ${ }^{18}$ C.A.N. Conde, ${ }^{12}$ A.A. Denisenko, ${ }^{1}$ G. Díaz, $,{ }^{2}, 14$ J. Díaz, ${ }^{17}$ M. Diesburg, ${ }^{5}$ J. Escada, ${ }^{12}$ R. Esteve,${ }^{20}$ R. Felkai, ${ }^{17}$ A.F.M. Fernandes, ${ }^{11}$ L.M.P. Fernandes, ${ }^{11}$ P. Ferrario, ${ }^{14,8}$ A.L. Ferreira, ${ }^{4}$ F.W. Foss Jr., ${ }^{1}$ E.D.C. Freitas, ${ }^{11}$ J. Generowicz, ${ }^{14}$ A. Goldschmidt, ${ }^{7}$ D. González-Díaz, ${ }^{2}$ J.J. Gómez-Cadenas, ${ }^{14,8, b}$ R. Guenette, ${ }^{10}$ R.M. Gutiérrez, ${ }^{9}$ J. Haefner, ${ }^{10}$ K. Hafidi, ${ }^{19}$ J. Hauptman, ${ }^{3}$ C.A.O. Henriques, ${ }^{11}$ J.A. Hernando Morata, ${ }^{2}$ P. Herrero, ${ }^{14,17}$ V. Herrero, ${ }^{20}$ S. Johnston, ${ }^{19}$ M. Kekic, ${ }^{17}$ L. Labarga, ${ }^{16}$ A. Laing, ${ }^{1}$ P. Lebrun, ${ }^{5}$ N. López-March,,${ }^{17}$ M. Losada, ${ }^{9}$ R.D.P. Mano,,${ }^{11}$ J. Martín-Albo, ${ }^{10}$ A. Martínez, ${ }^{14}$ G. Martínez-Lema, ${ }^{2,17}$ F. Monrabal, ${ }^{14}$ C.M.B. Monteiro, ${ }^{11}$ F.J. Mora, ${ }^{20}$ J. Muñoz Vidal, ${ }^{17}$ P. Novella, ${ }^{17}$ D.R. Nygren, ${ }^{1, c}$ B. Palmeiro, ${ }^{17}$ A. Para, ${ }^{5}$ J. Pérez, ${ }^{17, d}$ M. Querol, ${ }^{17}$ J. Renner, ${ }^{17}$ J. Repond, ${ }^{19}$ S. Riordan, ${ }^{19}$ L. Ripoll, ${ }^{15}$ Y. Rodriguez Garcia, ${ }^{9}$ J. Rodríguez, ${ }^{20}$ L. Rogers, ${ }^{1}$ B. Romeo, ${ }^{14}$ C. Romo-Luque, ${ }^{17}$ F.P. Santos, ${ }^{12}$ J.M.F. dos Santos, ${ }^{11}$ A. Simón, ${ }^{6}$ C. Sofka, ${ }^{13, e}$ M. Sorel, ${ }^{17}$ T. Stiegler, ${ }^{13}$ P. Thapa, ${ }^{1}$ J.F. Toledo, ${ }^{20}$ J. Torrent, ${ }^{14}$ A. Usón, ${ }^{17}$ J.F.C.A. Veloso, ${ }^{4}$ R. Webb, ${ }^{13}$ R. Weiss-Babai, ${ }^{6}$ J.T. White, ${ }^{13, f}$ N. Yahlali ${ }^{17}$

${ }^{1}$ Department of Physics, University of Texas at Arlington, Arlington, TX 76019, USA

${ }^{2}$ Instituto Gallego de Física de Altas Energías, Univ. de Santiago de Compostela, Campus sur, Rúa Xosé María Suárez Núñez, s/n, Santiago de Compostela, E-15782, Spain

${ }^{3}$ Department of Physics and Astronomy, Iowa State University, 12 Physics Hall, Ames, IA 50011-3160, USA

${ }^{4}$ Institute of Nanostructures, Nanomodelling and Nanofabrication (i3N), Universidade de Aveiro, Campus de Santiago, Aveiro, 3810-193, Portugal

${ }^{5}$ Fermi National Accelerator Laboratory, Batavia, IL 60510, USA

${ }^{6}$ Nuclear Engineering Unit, Faculty of Engineering Sciences, Ben-Gurion University of the Negev, P.O.B. 653, Beer-Sheva, 8410501, Israel

${ }^{7}$ Lawrence Berkeley National Laboratory (LBNL), 1 Cyclotron Road, Berkeley, CA 94720, USA

${ }^{8}$ Ikerbasque, Basque Foundation for Science, Bilbao, E-48013, Spain

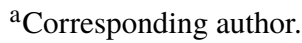

${ }^{b}$ NEXT Co-spokesperson.

${ }^{\mathrm{c}}$ NEXT Co-spokesperson.

${ }^{\mathrm{d}}$ Now at Laboratorio Subterráneo de Canfranc, Spain.

${ }^{\mathrm{e}}$ Now at University of Texas at Austin, USA.

${ }^{\mathrm{f}}$ Deceased.
} 
${ }^{9}$ Centro de Investigación en Ciencias Básicas y Aplicadas, Universidad Antonio Nariño, Sede Circunvalar, Carretera 3 Este No. 47 A-15, Bogotá, Colombia

${ }^{10}$ Department of Physics, Harvard University, Cambridge, MA 02138, USA

${ }^{11}$ LIBPhys, Physics Department, University of Coimbra, Rua Larga, Coimbra, 3004-516, Portugal

${ }^{12}$ LIP, Department of Physics, University of Coimbra, Coimbra, 3004-516, Portugal

${ }^{13}$ Department of Physics and Astronomy, Texas A\&M University, College Station, TX 77843-4242, USA

${ }^{14}$ Donostia International Physics Center (DIPC), Paseo Manuel Lardizabal, 4, Donostia-San Sebastian, E-20018, Spain

${ }^{15}$ Escola Politècnica Superior, Universitat de Girona, Av. Montilivi, s/n, Girona, E-17071, Spain

${ }^{16}$ Departamento de Física Teórica, Universidad Autónoma de Madrid, Campus de Cantoblanco, Madrid, E-28049, Spain

${ }^{17}$ Instituto de Física Corpuscular (IFIC), CSIC \& Universitat de València, Calle Catedrático José Beltrán, 2, Paterna, E-46980, Spain

${ }^{18}$ Pacific Northwest National Laboratory (PNNL), Richland, WA 99352, USA

${ }^{19}$ Argonne National Laboratory, Argonne, IL 60439, USA

${ }^{20}$ Instituto de Instrumentación para Imagen Molecular (I3M), Centro Mixto CSIC - Universitat Politècnica de València, Camino de Vera s/n, Valencia, E-46022, Spain

${ }^{21}$ Laboratorio de Física Nuclear y Astropartículas, Universidad de Zaragoza, Calle Pedro Cerbuna, 12, Zaragoza, E-50009, Spain

E-mail: katherine.woodruff@uta.edu

AbSTRACT: Motivated by the possibility of guiding daughter ions from double beta decay events to single-ion sensors for barium tagging, the NEXT collaboration is developing a program of R\&D to test radio frequency $(\mathrm{RF})$ carpets for ion transport in high pressure xenon gas. This would require carpet functionality in regimes at higher pressures than have been previously reported, implying correspondingly larger electrode voltages than in existing systems. This mode of operation appears plausible for contemporary RF-carpet geometries due to the higher predicted breakdown strength of high pressure xenon relative to low pressure helium, the working medium in most existing RF carpet devices. In this paper we present the first measurements of the high voltage strength of xenon gas at high pressure and at the relevant RF frequencies for ion transport (in the $10 \mathrm{MHz}$ range), as well as new DC and RF measurements of the breakdown strengths of high pressure argon and helium gases at small gap sizes. We find breakdown voltages that are compatible with stable RF carpet operation given the gas, pressure, voltage, materials and geometry of interest.

KEYwords: Gaseous detectors, Noble element TPCs 


\section{Contents}

1 Introduction 1

2 Experiment 3

2.1 Gas System 3

2.2 Electrode Configuration 4

2.3 HV Delivery and Monitoring System 4

2.4 Estimation of Systematic Uncertainty 5

3 Results and Discussion $\quad 6$

3.1 Comparison with Existing Data

3.2 Implications for RF Carpets in High Pressure Xenon 9

4 Conclusions 10

\section{Introduction}

Radio frequency (RF) charged particle manipulation techniques have been successfully used to trap and control ions for several decades [1]. As these techniques are becoming more efficient, they are being applied to a wider range of applications and environments. For example, RF carpets [2] have been shown to efficiently transport ions in a helium buffer gas for substantial distances in pressures up to 300 mbar [3-5]. Based on our still preliminary simulations, it appears that they could also be operated efficiently in pressures as high as 10 bar. In order to achieve stable motion, the higher pressure can be compensated for with higher RF voltages and smaller electrode spacing [6].

The RF voltage and electrode spacing in most devices using RF carpets is limited by dielectric breakdown of the buffer gas. The breakdown voltage is commonly described in terms of a Paschen curve [7], a supposedly universal function of the pressure times the gap distance for each gas. We expect the breakdown voltage to be significantly higher than achievable in helium, and with a beneficial scaling with pressure. There is also evidence that Paschen's law is frequency dependent [8]. The RF voltage breakdown strength in high pressure xenon gas at sub-millimeter distances has not been experimentally measured previously.

Our study of RF voltages in noble gases is motivated by the search for neutrinoless double beta decay in ${ }^{136} \mathrm{Xe}$. Observation of neutrinoless double beta decay $(0 v \beta \beta)$ would prove that neutrinos are Majorana fermions, that lepton number is not conserved, and could explain the matter-antimatter imbalance in the universe through leptogenesis [9]. If $0 v \beta \beta$ does exist, the expected rate would be very small and detection will require ton-scale detectors with unprecedentedly low background rates. Since known background processes induce nuclear $\mathrm{Z}+2$ transitions at an insignificant rate, a background free measurement could be achieved with the identification of the double beta decay daughter nucleus. ${ }^{136} \mathrm{Xe}$ is a common nuclide in neutrinoless double beta decay searches, and 
there are currently several ongoing efforts to identify the single barium ions that result from their decay [10-19].

For any of these barium tagging proposals to be successful, new technology will need to be developed to identify the barium ion within, or extract it from, the active volume of the xenon detector. In the case of high pressure gaseous xenon (HPGXe) TPCs, used by the NEXT collaboration [20], the barium ions are expected to be highly ionized initially, but will then undergo partial neutralization until they reach the doubly ionized state $\left(\mathrm{Ba}^{++}\right)$. The ion is slowly transported in various solvation states to the cathode via the drift electric field [21, 22]. Transport along the cathode to a small scanning region could be achieved using RF carpet ion manipulation techniques. Concepts based on RF have been previously explored in the context of double beta decay by the nEXO collaboration, most notably in [23]. There, an RF funnel was used to concentrate ions from a fast gas flow into an orifice for extraction to a vacuum ion trap. The RF carpet concept favored by the NEXT collaboration does not involve gas flow, but rather ion transport lateral to the cathode surface, leading the ion to an internal sensor operating in the high pressure gas.

Validity of the concept of RF carpet transport in high pressure xenon relies critically on being able to achieve sufficiently high breakdown voltages to drive the ions without significant losses. Preliminary simulations studies [24] indicate that we can transport $\mathrm{Ba}^{++}$in a xenon buffer gas at 10 bar with RF amplitudes as low as a few hundred volts with sub-millimeter electrode spacings. This would be significantly above what is typically realized in stopper cells, where dielectric breakdown of the low pressure helium buffer gas is often limiting above approximately 100V [25].

While there are no existing RF breakdown measurements in the pressure $(P)$ and gap distance (d) regions of interest, there have been RF and DC measurements near the $P \times d$ region, a universal combination under the Paschen formalism. DC breakdown in low pressure argon gas was reported in Refs. [25-28] in the $P \times d$ range up to 10 Torr $\mathrm{cm}$ with gap distances of several $\mathrm{cm}$. Systematic studies of the effect of electrode material and stressed area on DC breakdown in argon in the $P \times d$ region were carried out in [27]. RF ( $f=13.56 \mathrm{MHz})$ breakdown in low pressure argon was measured in Refs. [29-31] up to 30 Torr $\mathrm{cm}$ also with gap distances of several $\mathrm{cm}$. In low pressure xenon, measurements of the DC breakdown voltage in $P \times d$ range including 1 to 10 Torr $\mathrm{cm}$ were reported in Refs. [32, 33], and the voltage breakdown at microwave frequencies $(f=2800 \mathrm{MHz})$ covering the same $P \times d$ range was measured in Ref. [34]. In low pressure helium, RF ( $f=13.56 \mathrm{MHz}$ ) voltage breakdown was measure covering the same $P \times d$ range in Refs. [35, 36] with gap distances as small as $1 \mathrm{~mm}$.

In this paper, we describe our measurements of the Paschen curve for DC and RF voltages in the pressure and electrode gap ranges of interest for operating RF carpets in high pressure gases. In addition to xenon, we measured the breakdown voltages in argon and helium (which are more commonly used as RF carpet buffer gases) in pressures from 100 mbar to 10 bar. We show in argon that tripling the electrode gap distance from $5 \mathrm{mil}(0.127 \mathrm{~mm})$ to $15 \mathrm{mil}(0.381 \mathrm{~mm})$ has no significant effect on the breakdown voltage at fixed $P \times d$, so we only include the 5 mil gap in the helium and xenon measurements. All of the RF measurements are performed at a frequency of 13.56 MHz. It has been shown in Ref. [37] that in the low MHz frequency regime, the efficiency of RF carpet transport increases with increasing frequency up to approximately $10 \mathrm{MHz}$ at which point the efficiency stays constant, and so $13.56 \mathrm{MHz}$ is chosen to be centrally within our range of interest. 
In Sec. 2 we describe the experiment, including the electrode configuration in 2.2, the DC and RF delivery and monitoring systems in 2.3 , the gas handling system in 2.1, and the data taking methodology including our estimations of systematic uncertainties in 2.4.

\section{Experiment}

The experimental setup, shown in Fig. 1, consists of two electrodes connected to (RF or DC) high voltage (HV) inside of a pressure vessel capable of holding pressures up to 10 bar. For each gas, three breakdown measurements are taken per pressure. When the vessel is at the desired gas pressure, the RF or DC high voltage is increased until breakdown is observed. For the RF breakdown measurements, the voltage is monitored through an oscilloscope, described in Sec. 2.3. When breakdown occurs, the RF circuit goes out of resonance, and the signal on the oscilloscope collapses. For the DC breakdown measurements, the voltage is monitored on the DC power supply itself. When DC breakdown occurs, the voltage drops and the current increases until there is a current trip in the power supply. For both RF and DC, the highest voltage observed before the breakdown is recorded as the breakdown voltage.

\subsection{Gas System}

The electrodes are housed inside a five-liter pressure vessel connected to a gas handling and circulation system. The same gas system was used in previous studies of Refs [38, 39], where further information can be found. The gases used are ultra-high purity grade helium and argon from AirGas, and xenon from Concorde Specialty Gases. Before each gas fill, the system is evacuated to better than $10^{-5}$ mbar pressure. The xenon is filled through a MonoTorr PS-4-MT3 hot getter to the maximum pressure and circulated using a MetroCad PumpWorks PW-2070 pump with flow rates between one and ten liters per minute until the pressure vessel volume has been circulated through the getter ten times. The process is the same for the helium and argon gases, except they are filled and circulated through a Saes PureGas MicroTorr HP190-902-FV cold getter instead of the hot getter. HV breakdown measurements are taken at the highest pressure, then at discrete steps down in pressure as the gas is either vented into a separate evacuated vessel (in the case of helium and argon) or reclaimed (in the case of xenon). The gas pressure in the vessel is monitored with a pressure transducer with 10 mbar precision.

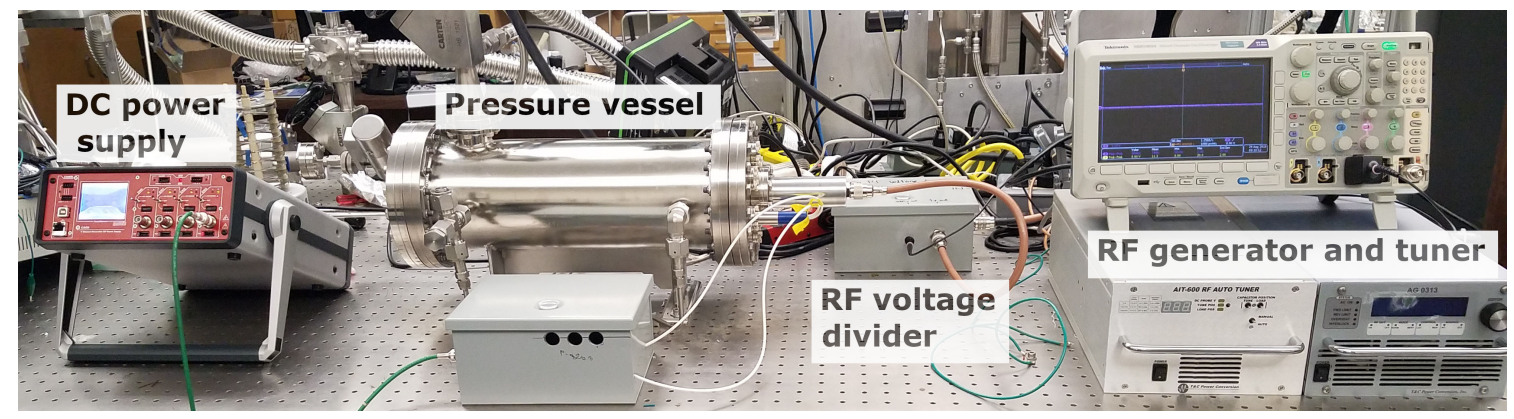

Figure 1. Photograph of the experimental setup. 


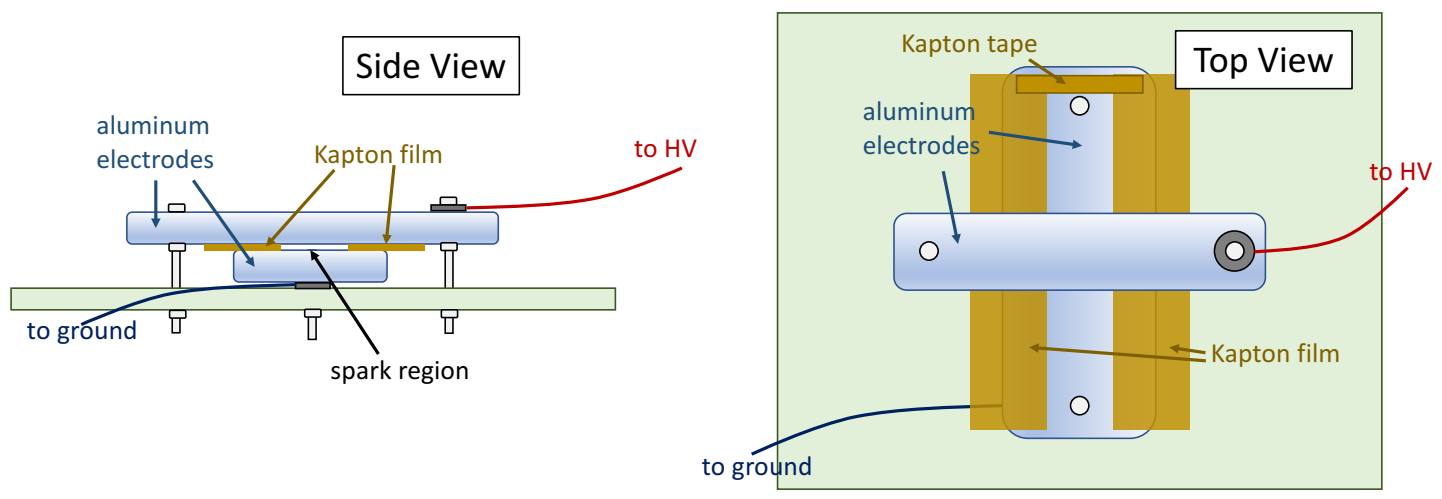

Figure 2. Diagram of the electrode configuration.

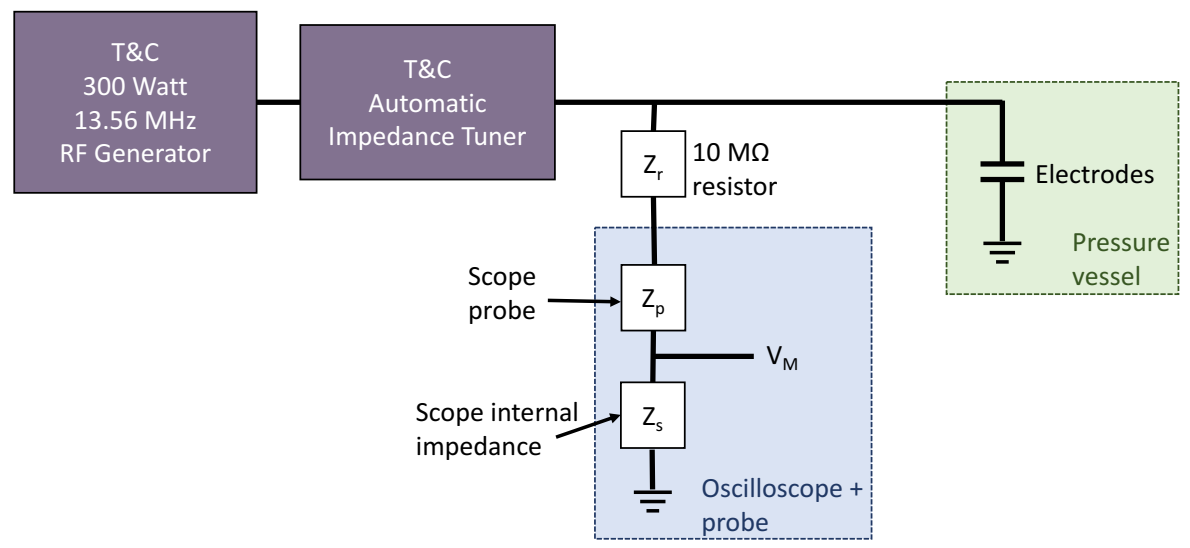

Figure 3. Diagram of the RF circuit.

\subsection{Electrode Configuration}

The electrodes consist of two aluminum bars positioned in a cross, as shown in Fig. 2. The top bar is $2.5 \mathrm{in}$. $(63.5 \mathrm{~mm})$ long by $1.0 \mathrm{in}$. $(25.4 \mathrm{~mm})$ wide, and the bottom bar is $2.5 \mathrm{in}$. $(63.5 \mathrm{~mm})$ long by $0.5 \mathrm{in}$. $(12.7 \mathrm{~mm})$ wide. The cross configuration is used to reduce the amount of electrically stressed area, which has been shown to affect the breakdown voltage [40]. The top bar is connected to the HV signal, and the bottom bar is connected to ground. Two pieces of thin, 5 mil (0.005 in. or $0.127 \mathrm{~mm}$ ) Kapton film, $0.375 \mathrm{in}$. $(9.525 \mathrm{~mm})$ apart, are sandwiched in between the electrodes to set the gap distance. The voltage breakdown generally happens in the open area between the electrodes where no Kapton is placed. The total open (stressed) area between the electrodes is $0.1875 \mathrm{in.}^{2}\left(121 \mathrm{~mm}^{2}\right)$. The entire apparatus is held in place with nylon screws onto a large (4 in. or $102 \mathrm{~mm}$ ) piece of HDPE.

\subsection{HV Delivery and Monitoring System}

The RF HV is supplied by a $13.56 \mathrm{MHz}, 300$-watt AG Plasma Series generator and an automatic impedance tuner from $\mathrm{T} \& \mathrm{C}$ Power Conversion. The RF voltage is actively monitored through 

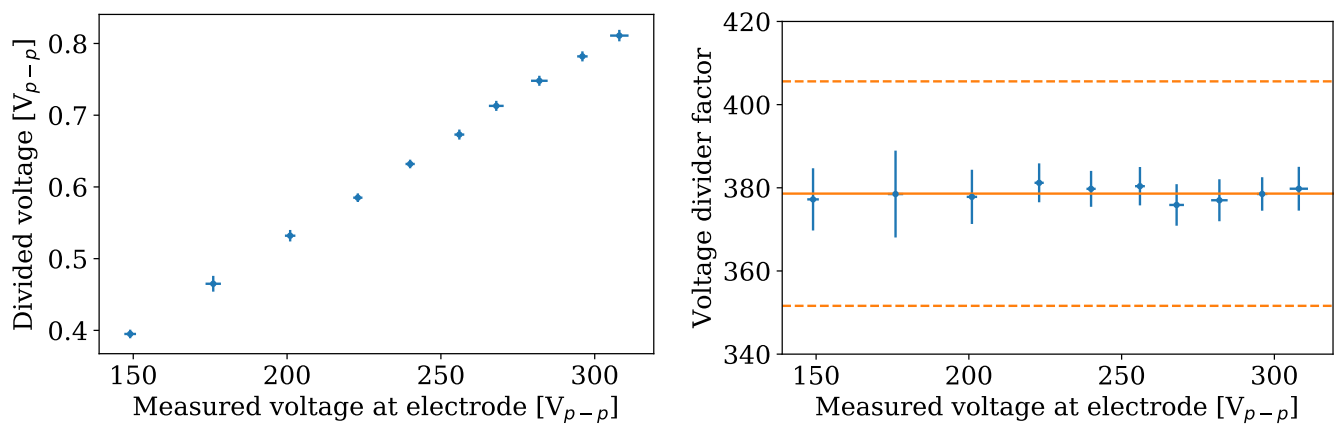

Figure 4. Example voltage divider measurement. The left plot shows the comparison of the peak-to-peak voltage measured at the electrode and the peak-to-peak voltage measured after the potential divider with the uncertainty on the voltage measurement. The right plot shows the ratio of the two. The error bars on the points show the uncertainty on the peak voltages measured at the electrode and after the potential divider. The solid line in the right plot shows the average voltage divider factor, and the dashed lines show the combined (one standard deviation) uncertainty on the true voltage divider factor.

a potential divider to an oscilloscope, as shown in Fig. 3. The potential divider consists of a $10 \mathrm{M} \Omega$ resistor (labeled $Z_{r}$ ), the oscilloscope probe (labeled $Z_{p}$ ), and the internal impedance of the oscilloscope itself (labeled $Z_{s}$ ). The impedance of each component at $13.56 \mathrm{MHz}$ is not precisely known, but the voltage division factor can be measured. We found that the exact voltage division factor varied with different electrode spacings and impedance tuner settings, presumably due to changing capacitances and inductances within the circuit impacting the impedance at radio frequencies. Therefore, it was measured directly for each configuration. Fig. 4 shows an example measurement of the voltage divider factor for one electrode configuration. The left plot shows the peak-to-peak voltage measured after the potential divider as a function of the peak-to-peak voltage measured at the HV electrode, and the right plot shows the voltage divider factor with the total uncertainty on the conversion to true voltage at the HV electrode. The RF signal is delivered to the electrodes inside a pressure chamber through a CeramTec copper RF power feedthrough. The DC HV is supplied by a CAEN DT1471HET desktop HV power supply.

\subsection{Estimation of Systematic Uncertainty}

The sources of systematic uncertainty that we took into account are the uncertainty in the gas pressure measurement, the uncertainty in the spacing between the electrodes, and the uncertainty in the voltage measurement. We also tested the effect on the breakdown voltage due to the electrode material and the size of the stressed area.

The uncertainty in the gas pressure measurement is 10 mbar and is due to the precision of the transducer. The uncertainty on the gap distance is due to the compression of the Kapton film between the electrodes and the flatness of the electrodes. Based on the large elastic modulus of Kapton and absence of any observable electrode curvature when compared to a granite surface plate, the uncertainty in the gap distance is estimated to be less than $10 \%$.

The uncertainty in the breakdown voltage is due to the measurement of the voltage at the electrode and to irreducible error from the randomness in the breakdown process itself. The uncertainty in the measurement is estimated from the variance in the voltage division factor, 
described in Sec. 2.3, and in the change in the voltage measured at the HV electrode using different, compensated, oscilloscope probes, which is found to be 5\%. To account for the irreducible error, several measurements were taken consecutively at each pressure, and the standard deviation of the measured breakdown voltages was added in quadrature to the uncertainty on the voltage measurement.

\section{Results and Discussion}

Fig. 5 shows the measured RF and DC breakdown voltages in helium, argon, and xenon with a 5 mil gap distance. In the case of both xenon and argon gases, breakdown through the Kapton spacer ultimately became limiting at a field strength of $40 \mathrm{kV} / \mathrm{cm}$. Before breakdown through the Kapton became prevalent, inspection of traces on the electrode surfaces showed breakdown was across the gas gap, rather than along the insulating surface. All gases show the expected Paschen-like increase of HV strengh with increasing pressure under both RF and DC. The RF breakdown voltages are found to be systematically lower than the DC ones, an effect which is most significant for xenon gas, and represents an approximately $30 \%$ effect.

When designing this test, we investigated several possible variables that may correlate with breakdown strength, with results shown in Fig. 6. To determine whether electrode material is significantly correlated with breakdown strength, the breakdown voltage in argon was measured with copper as well as aluminum electrodes. The work functions of these metals are sufficiently different (4.53-5.10 eV for $\mathrm{Cu}$ compared to $4.06-4.26 \mathrm{eV}$ for $\mathrm{Al}$ ) that any substantial dependence through photoelectron emission would likely be revealed. Fig. 6 shows that there is some systematic

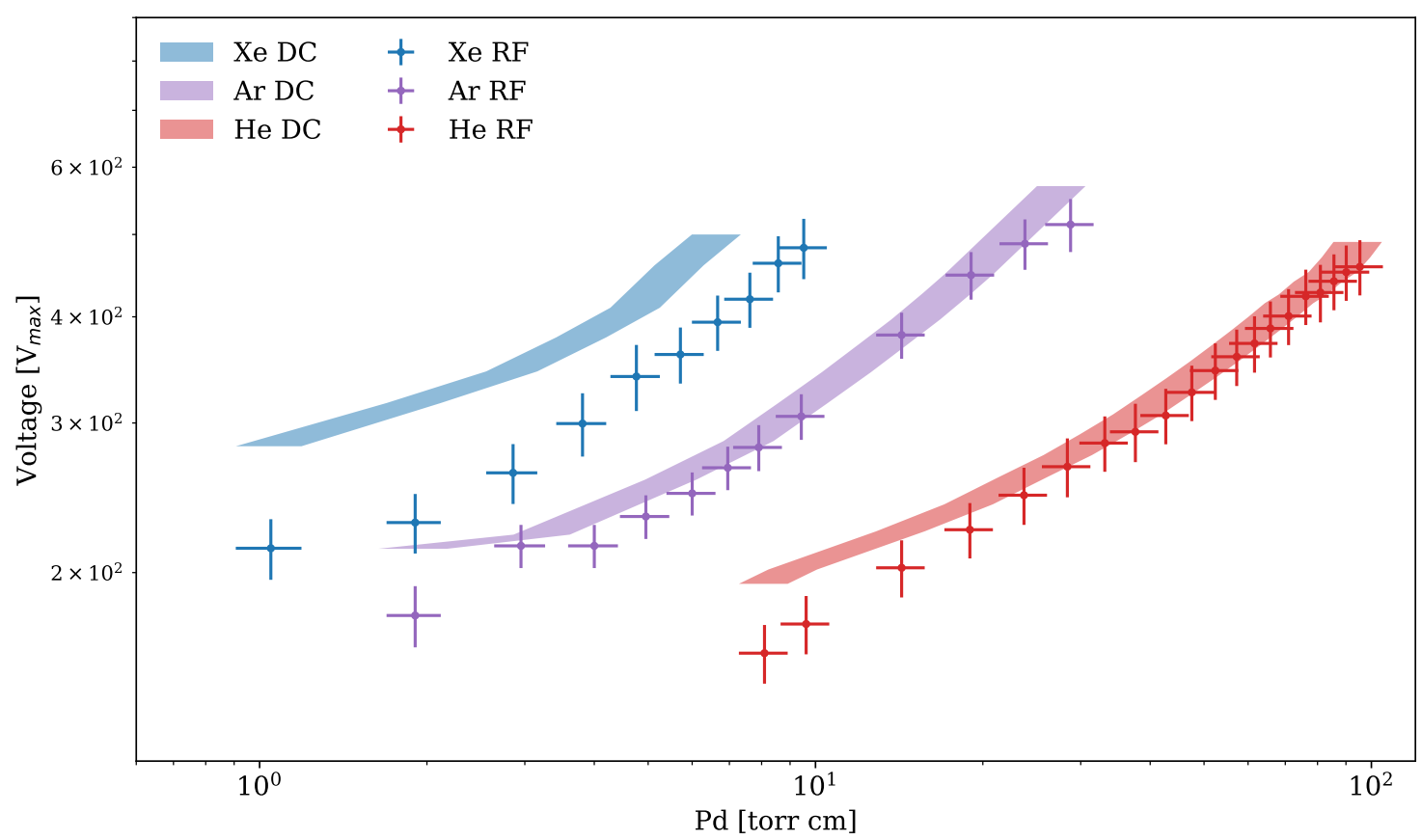

Figure 5. RF and DC breakdown voltages in xenon, argon, and helium using aluminum electrodes with a 5 mil $(0.127 \mathrm{~mm})$ gap. The RF voltage shown is the maximum amplitude of the RF signal. 


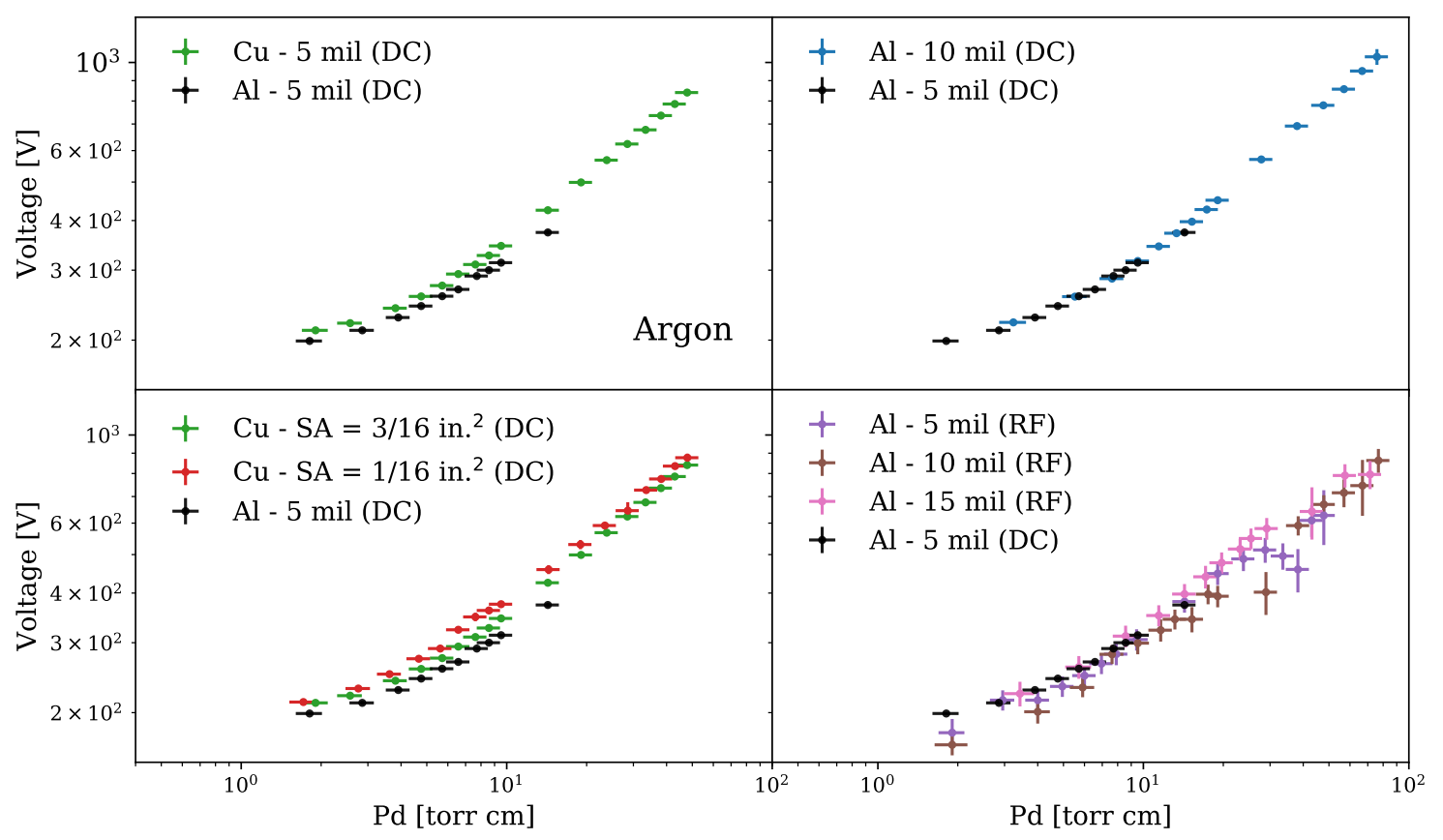

Figure 6. Comparison of breakdown voltages in argon using different electrode configurations. The top left plot shows the effect of using different electrode materials, the bottom left plot shows the effect of different stressed areas, the top right plots show the effect of different gap distances with DC on the top and RF on the bottom.

trend, but it is rather small, barely larger than systematic uncertainty. We tested dependence on stressed area using similar electrodes with a larger Kapton insulator resulting in 1/3 of the original electrode surface available for breakdown. The results of these tests are shown in Fig. 6 lower left. Again, a small effect is observed, but it appears consistent with the scale of systematic uncertainty. We also tested Paschen scaling by changing the gap size. We observed that changing the gap distance and pressure had no significant effect on either the RF of DC breakdown voltages at fixed $P \times d$, validating Paschen-like behaviour in this regime. These comparisons are shown for both RF and DC voltages in right panels of Fig. 6.

\subsection{Comparison with Existing Data}

Fig. 7 shows the comparisons of the DC (left) and RF (right) breakdown voltages in high pressure argon to a selection of existing measurements in low pressure argon in the same $P \times d$ range. In both DC and RF, the measurements fall in the middle of the spread of existing data. The behavior of the comparison between the different electrode materials and stressed areas form Farag et al. in Fig. 7 left agree well with the behavior shown in Fig. 6.

Fig. 8 shows the comparisons of the DC and RF breakdowns in xenon (left) and helium (right) to a selection of existing data. The previous existing data were taken with gap distances in the range of 6-32 $\mathrm{mm}$ for xenon and $0.4-10 \mathrm{~mm}$ for helium at sub-atmospheric pressures compared to ours, taken at a 5 mil $(0.127 \mathrm{~mm})$ gap distance. The DC breakdown measurements (shaded area) agree very well with existing data, and the RF breakdown measurements fall between the existing DC and 

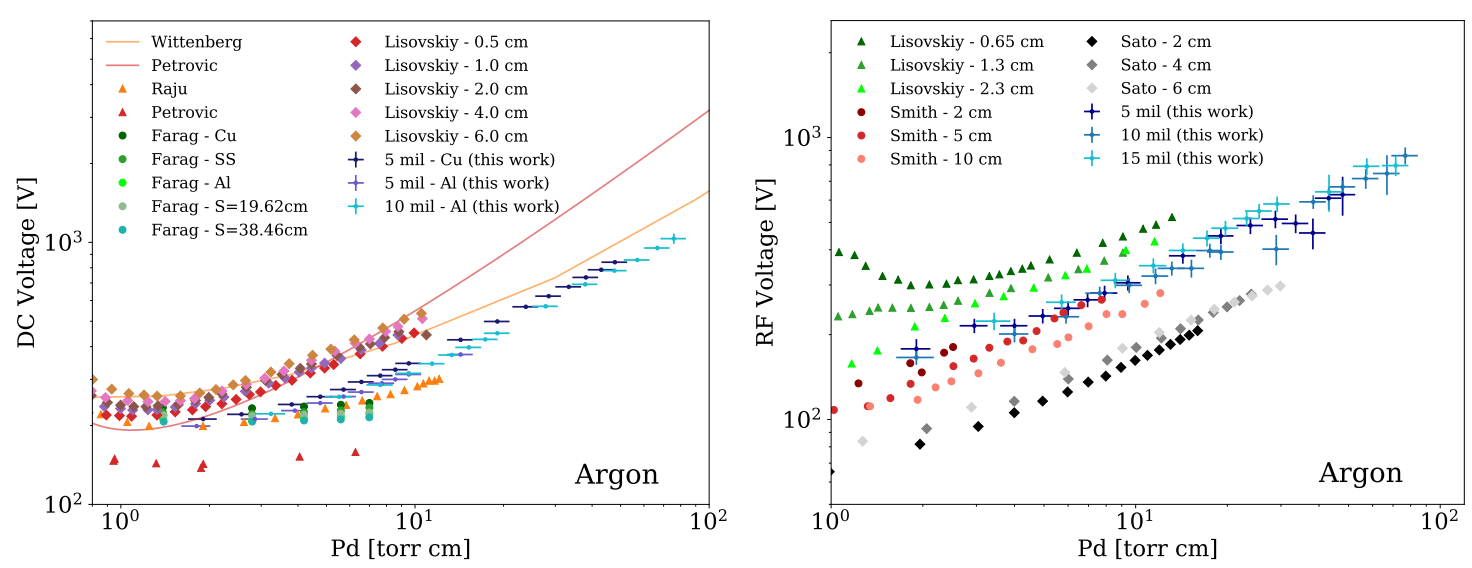

Figure 7. Comparison of DC (left) and RF (right) voltage breakdown in argon gas to a selection of existing data. The RF measurements were all taken at $13.56 \mathrm{MHz}$, and the maximum voltage amplitude is reported. In the left plot, the triangles are reported in textbooks [25] and [26], the circles [27] represent different electrode materials ( $\mathrm{Cu}, \mathrm{Al}$, and stainless steel) and different stressed areas $(19.62 \mathrm{~cm}$ and $38.46 \mathrm{~cm})$ with Cu electrodes. The diamonds were measured in Ref. [28]. The lines are the parameterized curves in Refs. [25, 41]. In the right plot, the triangles were measured in Ref. [29], the circles in Ref. [30], and the diamonds in Ref. [31].
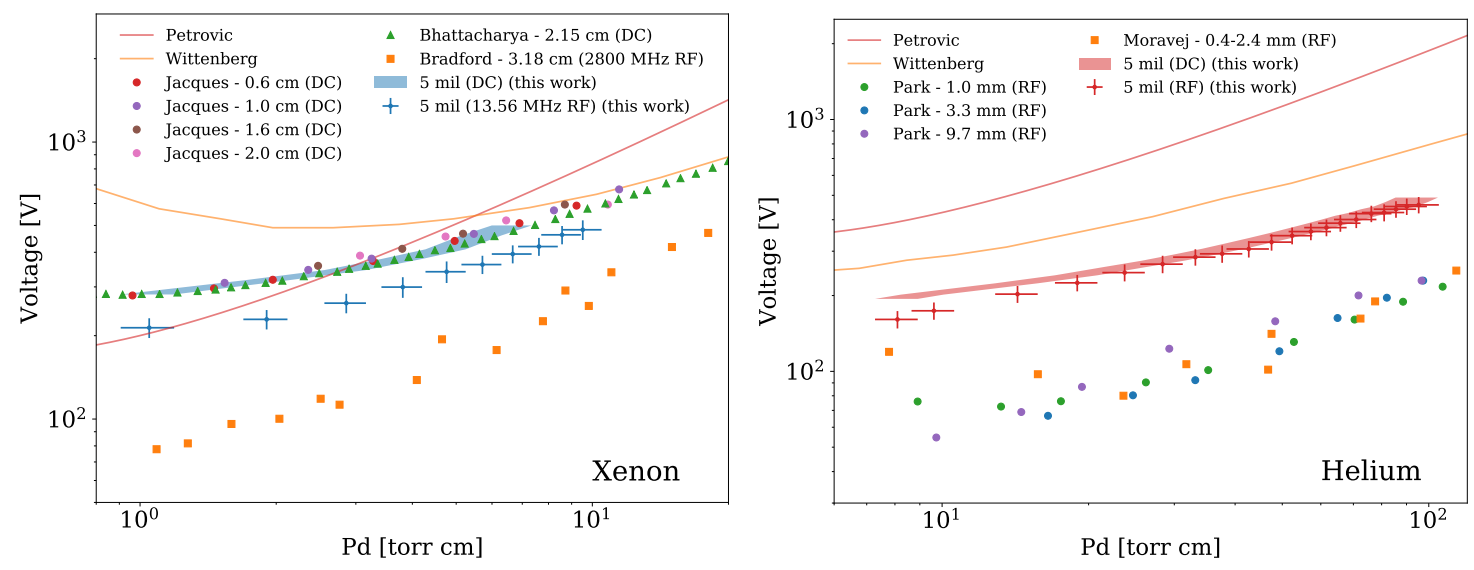

Figure 8. Comparison of xenon (left) and helium (right) RF and DC voltage breakdown measurements to a selection of existing data. The DC measurements in this work are shown as shaded area in both plots, and the RF measurements are shown as blue points with error bars for xenon and red points with error bars for helium. In the left plot, the DC circles [33] were measured using gold electrodes, and the DC triangles [32] were measured using nickel electrodes, and the squares [34] represent microwave breakdown measurements at $2800 \mathrm{MHz}$. In the right plot, the circles [35] were measured with aluminum electrodes, and the squares [36] were measured with several gap distances from $0.4-2.4 \mathrm{~mm}$. The lines are the parameterized curves in Refs. [25, 41].

microwave breakdown measurements. Both the helium RF and DC measurements in Fig. 8 right fall well above the existing RF measurements also taken at $13.56 \mathrm{MHz}$.

Fig. 9 shows the agreement between our DC and RF breakdown measurements in argon and xenon and high pressure, large gap-size DC breakdown measurements performed previously by this group described in Ref. [39]. The previous measurements were taken at similar pressures within 


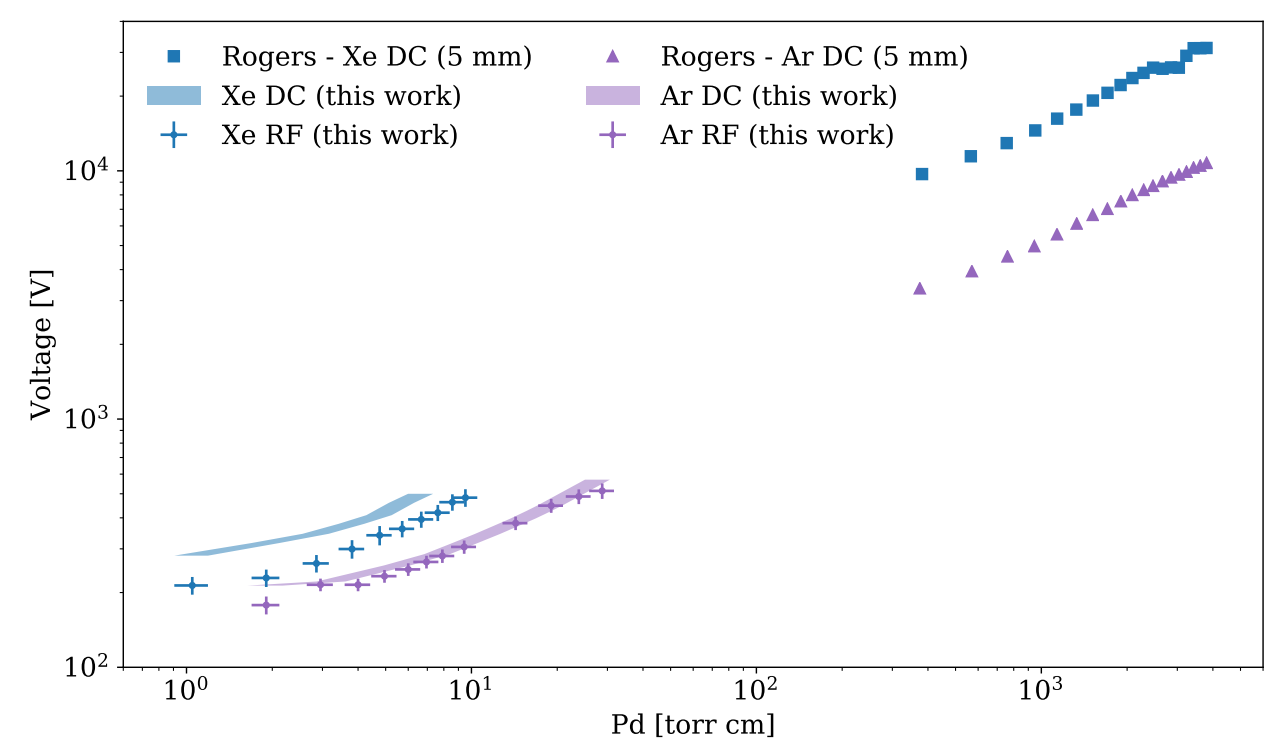

Figure 9. Xenon and argon RF and DC voltage breakdown measurements from this work with previous high pressure xenon and argon DC breakdown measurements with larger gap distance from Ref. [39]. .

the same gas system, with gap distances of $5 \mathrm{~mm}$.

\subsection{Implications for RF Carpets in High Pressure Xenon}

The pressure ranges where in-gas breakdowns could be studied were 1 to 10 bar in helium, 0.2 to 4 bar in argon, and 0.1 to 1 bar in xenon. Since RF carpets with small electrode pitches are commonly printed on a Kapton substrate, our data strongly suggest that the Kapton will be the limiting factor in their operation in xenon at 10 bar, rather than through-gas breakdowns, as are limiting in the case of low pressure helium. For a 5 mil pitch carpet, the threshold of operation may thus be close to $500 \mathrm{~V}$ peak-to-peak. It is notable, however, that in such a carpet the copper electrode traces are laid on top of the Kapton and not on either side as in the configuration here. This implies a smaller electric field through the Kapton itself, and thus our measurements here may be considered as a cautious lower limit. Future voltage breakdown tests of printed copper electrode traces on a "flex" Kapton board will determine if this is true.

Comparing this experimental constraint with our simulations, we observe that significant parameter space remains open where RF carpet operation is theoretically predicted to be possible in xenon gas at 10 bar. Preliminary simulations have shown that $\mathrm{Ba}^{++}$ion transport in xenon buffer gas at 10 bar should be achievable with RF voltages as low as $250 \mathrm{~V}$, given existing electrode pitch geometries with 4 mil $(0.1 \mathrm{~mm})$ gap distances.

The next phase of our research program will be to demonstrate ion transport using a $5 \mathrm{~cm}$ scale RF carpet in xenon gas. The results of this test will help establish the viability of RF-carpet based ion guiding schemes for barium tagging within high pressure xenon gas detectors. 


\section{Conclusions}

We have presented the first RF breakdown strength measurements in xenon gas at pressures above 100 Torr and at frequencies in the MHz range, as well as new measurements of RF and DC breakdown voltages in argon at pressures of 100 mbar-1 bar and helium at pressures of 1 bar-10 bar and sub-millimeter gap spacings.

We find that the RF breakdown voltages in high pressure helium, argon, and xenon for submillimeter gap distances agree approximately with existing low pressure data in the same $P \times d$ range with much larger gap distances. In the cases of argon and xenon in the $P \times d$ region between 10 and 100 Torr $\mathrm{cm}$, the RF and DC breakdown voltages agree well with each other. There are some deviations between the xenon RF and DC breakdown voltages, but these measurements all exist below 10 Torr $\mathrm{cm}$, where we start to observe deviations in the argon and helium as well. Based on our data, the limiting factor in the application to high pressure RF carpets is likely to be the Kapton substrate that starts to fail at approximately $500 \mathrm{~V}$ at 5 mil thickness, rather than the gas itself. This is distinct to the case of helium-based systems, where through-gas breakdowns are limitting. This warrants future tests of breakdown between electrodes printed on a Kapton substrate.

These results suggest significant promise for the concept of using RF carpets in high pressure xenon gas. Such an a device may prove to be a critical ingredient in a workable scheme for barium ion identification within xenon gas time projection chambers.

\section{References}

[1] HG Dehmelt and FG Major. Orientation of (he 4)+ ions by exchange collisions with cesium atoms. Physical Review Letters, 8(5):213, 1962.

[2] Michiharu Wada, Yoshihisa Ishida, Takashi Nakamura, Yasunori Yamazaki, Tadashi Kambara, Hitoshi Ohyama, Yasushi Kanai, Takao M Kojima, Youichi Nakai, Nagayasu Ohshima, et al. Slow ri-beams from projectile fragment separators. Nuclear Instruments and Methods in Physics Research Section B: Beam Interactions with Materials and Atoms, 204:570-581, 2003.

[3] Amanda Elizabeth Gehring, Maxime Brodeur, Georg Bollen, David J Morrissey, and Stefan Schwarz. Research and development of ion surfing rf carpets for the cyclotron gas stopper at the nscl. Nuclear Instruments and Methods in Physics Research Section B: Beam Interactions with Materials and Atoms, 376:221-224, 2016.

[4] Manisha Ranjan, S Purushothaman, T Dickel, H Geissel, WR Plass, D Schäfer, C Scheidenberger, J Van de Walle, H Weick, and P Dendooven. New stopping cell capabilities: Rf carpet performance at high gas density and cryogenic operation. EPL (Europhysics Letters), 96(5):52001, 2011.

[5] Fumiya Arai, Yuta Ito, Ichiro Katayama, Peter Schury, Tetsu Sonoda, Michiharu Wada, and Hermann Wollnik. Performance of ion surfing rf-carpets for high-energy ri beam gas catcher. In Proceedings of the Conference on Advances in Radioactive Isotope Science (ARIS2014), page 030110, 2015.

[6] S Schwarz. Rf ion carpets: The electric field, the effective potential, operational parameters and an analysis of stability. International Journal of Mass Spectrometry, 299(2-3):71-77, 2011.

[7] Friedrich Paschen. Ueber die zum funkenübergang in luft, wasserstoff und kohlensäure bei verschiedenen drucken erforderliche potentialdifferenz. Annalen der Physik, 273(5):69-96, 1889.

[8] WG Townsend and GC Williams. The electrical breakdown of gases in uniform high frequency fields at low pressure. Proceedings of the Physical Society, 72(5):823, 1958. 
[9] M Fukugita and T Yanagida. Baryogenesis without grand unification. Phys. Lett. B, 174:45, 1986.

[10] D Sinclair, E Rollin, J Smith, A Mommers, N Ackeran, B Aharmin, M Auger, PS Barbeau, C Benitez-Medina, M Breidenbach, et al. Prospects for barium tagging in gaseous xenon. Journal of Physics: Conference Series, 309(1):012005, 2011.

[11] B Mong, S Cook, T Walton, C Chambers, A Craycraft, C Benitez-Medina, K Hall, W Fairbank Jr, JB Albert, DJ Auty, et al. Spectroscopy of ba and ba+ deposits in solid xenon for barium tagging in nexo. Physical Review A, 91(2):022505, 2015.

[12] David R Nygren. Detection of the barium daughter in 136xe-> 136ba + 2e- by in situ single-molecule fluorescence imaging. Nuclear Instruments and Methods in Physics Research Section A: Accelerators, Spectrometers, Detectors and Associated Equipment, 824:2-5, 2016.

[13] BJP Jones, AD McDonald, and DR Nygren. Single molecule fluorescence imaging as a technique for barium tagging in neutrinoless double beta decay. Journal of Instrumentation, 11(12):P12011, 2016.

[14] N Byrnes, FW Foss Jr, BJ Jones, AD McDonald, DR Nygren, P Thapa, and A Trinidad. Progress toward barium tagging in high pressure xenon gas with single molecule fluorescence imaging. arXiv preprint arXiv:1901.03369, 2019.

[15] AD McDonald, BJP Jones, DR Nygren, C Adams, V Álvarez, CDR Azevedo, JM Benlloch-Rodríguez, FIGM Borges, A Botas, S Cárcel, et al. Demonstration of single-barium-ion sensitivity for neutrinoless double-beta decay using single-molecule fluorescence imaging. Physical review letters, 120(13):132504, 2018.

[16] C Chambers, T Walton, D Fairbank, A Craycraft, DR Yahne, J Todd, A Iverson, W Fairbank, A Alamare, JB Albert, et al. Imaging individual barium atoms in solid xenon for barium tagging in nexo. Nature, 569:203-207, 2018.

[17] P Thapa, I Arnquist, N Byrnes, AA Denisenko, FW Foss Jr, BJP Jones, AD McDonald, DR Nygren, and $\mathrm{K}$ Woodruff. Barium chemosensors with dry-phase fluorescence for neutrinoless double beta decay. Accepted by Nature Sci. Rep., 2019.

[18] N.K. Byrnes, A.A. Denisenko, F.W. Foss Jr., B.J.P. Jones, A.D. McDonald, D.R. Nygren, P. Thapa, $\mathrm{K}$. Woodruff, et al. Barium tagging with selective, dry-functional, single moleculesensitive on-off fluorophores for the next experiment. In Meeting of the Division of Particles and Fields of the American Physical Society, Northeastern University, Boston, 2019.

[19] Iván Rivilla, Borja Aparicio, Juan M. Bueno, David Casanova, Claire Tonnelé, Zoraida Freixa, Pablo Herrero, José I. Miranda, Rosa M. Martínez-Ojeda, Francesc Monrabal, Beñat Olave, Thomas Schafer, Pablo Artal, Fernando P. Cossío, Juan J. Gómez-Cadenas, et al. Towards a background-free neutrinoless double betadecay experiment based on a fluorescent bicolor sensor. arXiv preprint, 2019.

[20] Justo Martín-Albo, J Muñoz Vidal, P Ferrario, M Nebot-Guinot, JJ Gómez-Cadenas, V Álvarez, CDR Azevedo, FIG Borges, S Cárcel, JV Carrión, et al. Sensitivity of next-100 to neutrinoless double beta decay. Journal of High Energy Physics, 2016(5):159, 2016.

[21] E. Bainglass, B. J. P. Jones, F. W. Foss, M. N. Huda, and D. R. Nygren. Mobility and Clustering of Barium Ions and Dications in High Pressure Xenon Gas. Phys. Rev., A97(6):062509, 2018.

[22] P. Novella et al. Measurement of radon-induced backgrounds in the NEXT double beta decay experiment. Journal of High Energy Physics, 10:112, 2018.

[23] Thomas Brunner, Daniel Fudenberg, Victor Varentsov, Amanda Sabourov, Giorgio Gratta, Jens Dilling, Ralph DeVoe, David Sinclair, William Fairbank Jr, Joshua B Albert, et al. An rf-only 
ion-funnel for extraction from high-pressure gases. International Journal of Mass Spectrometry, 379:110-120, 2015.

[24] K Woodruff. Barium ion transport in high pressure xenon gas using rf carpets. Presented at the 13th International Conference on Stopping and Manipulation of Ions, McGill University, July 2019.

[25] Michael A Lieberman and Alan J Lichtenberg. Principles of plasma discharges and materials processing. John Wiley \& Sons, 2005.

[26] Merle Hirsh. Gaseous electronics, volume 1. Elsevier, 2012.

[27] Omar F Farag, Magdy M Mansour, Naglaa M El-Sayed, and Mohammed H Elghazaly. Mixing and cathode material effects on breakdown voltage of dc ar glow discharge. Advances in Applied Science Research, 4(1):146-150, 2013.

[28] VA Lisovskiy, SD Yakovin, and VD Yegorenkov. Low-pressure gas breakdown in uniform dc electric field. Journal of Physics D: Applied Physics, 33(21):2722, 2000.

[29] VA Lisovskiy and VD Yegorenkov. Rf breakdown of low-pressure gas and a novel method for determination of electron-drift velocities in gases. Journal of Physics D: Applied Physics, 31(23):3349, 1998.

[30] HB Smith, Christine Charles, and RW Boswell. Breakdown behavior in radio-frequency argon discharges. Physics of Plasmas, 10(3):875-881, 2003.

[31] Masumi Sato and Masafumi Shoji. Breakdown characteristics of rf argon capacitive discharge. Japanese journal of applied physics, 36(9R):5729, 1997.

[32] AK Bhattacharya. Measurement of breakdown potentials and townsend ionization coefficients for the penning mixtures of neon and xenon. Physical Review A, 13(3):1219, 1976.

[33] L Jacques, W Bruynooghe, R Boucique, and W Wieme. Experimental determination of the primary and secondary ionisation coefficients in krypton and xenon. Journal of Physics D: Applied Physics, 19(9):1731, 1986.

[34] HM Bradford, DM Fraser, GFO Langstroth, and AD MacDonald. Electrical breakdown in xenon and krypton at ultrahigh frequencies. Canadian Journal of Physics, 37(10):1166-1170, 1959.

[35] Jaeyoung Park, I Henins, HW Herrmann, and GS Selwyn. Gas breakdown in an atmospheric pressure radio-frequency capacitive plasma source. Journal of Applied Physics, 89(1):15-19, 2001.

[36] M Moravej, X Yang, GR Nowling, JP Chang, RF Hicks, and SE Babayan. Physics of high-pressure helium and argon radio-frequency plasmas. Journal of Applied Physics, 96(12):7011-7017, 2004.

[37] Gregory Keith Pang. Development of radiofrequency carpets for ion transport at the NSCL. 2011.

[38] A. D. McDonald et al. Electron Drift and Longitudinal Diffusion in High Pressure Xenon-Helium Gas Mixtures. Journal of Instrumentation, 14(08):P08009, 2019.

[39] L Rogers, RA Clark, BJP Jones, AD McDonald, DR Nygren, F Psihas, C Adams, V Álvarez, L Arazi, CDR Azevedo, et al. High voltage insulation and gas absorption of polymers in high pressure argon and xenon gases. Journal of Instrumentation, 13(10):P10002, 2018.

[40] M Okawa, T Shioiri, H Okubo, and S Yanabu. Area effect on electric breakdown of copper and stainless steel electrodes in vacuum. IEEE Transactions on Electrical Insulation, 23(1):77-81, 1988.

[41] HH Wittenberg. Gas tube design. Electron Tube Design, 792, 1962. 